CLINICAL STUDY

\title{
Autonomic nervous system balance in children and adolescents with craniopharyngioma and hypothalamic obesity
}

\author{
Michal Cohen ${ }^{1,3}$, Catriona Syme ${ }^{1,3}$, Brian W McCrindle ${ }^{2,3}$ and Jill Hamilton ${ }^{1,3}$ \\ ${ }^{1}$ Division of Endocrinology and ${ }^{2}$ Division of Cardiology, The Hospital for Sick Children, 555 University Avenue, Toronto, Ontario M5G1X8, Canada, \\ and ${ }^{3}$ The University of Toronto, Toronto, Ontario, Canada \\ (Correspondence should be addressed to J Hamilton at Division of Endocrinology, The Hospital for Sick Children; Email: jill.hamilton@sickkids.ca)
}

\begin{abstract}
Objective: Dysregulation of the autonomic nervous system is thought to be involved in craniopharyngioma-related hypothalamic obesity (CRHO). Increased parasympathetic activity and decreased sympathetic activity have been suggested. We aimed to study autonomic activity using heart rate variability (HRV) and biochemical measures in youth with CRHO compared with controls and to explore relationships between obesity and autonomic indices.

Design: A cross-sectional study of 16 youth with CRHO and 16 controls matched for sex, age, and BMI. Methods: Anthropometrics, fasting blood-work, resting energy expenditure (REE), 24-h HRV, and 24-h urine catecholamines were assessed. Quality of life, sleepiness, and autonomic symptoms were evaluated. Power spectral analysis of the HRV was performed.

Results: HRV power spectral analysis parameters of both parasympathetic activity (mean high frequency $\left(\mathrm{HF}\left(\mathrm{ms}^{2}\right)\right) 611 \pm 504$ vs $\left.459 \pm 336, P=0.325\right)$ and sympathetic activity (median low frequency/HF $1.62(1.37,2.41)$ vs $1.89(1.44,2.99), P=0.650)$ did not differ between the groups. Parasympathetic activity negatively correlated with central adiposity in both groups $(r=-0.53$, $P=0.034$ and $r=-0.54, P=0.029$ ) and sympathetic activity positively correlated with central adiposity in CRHO $(r=0.51, P=0.043)$. Youth with CRHO had significantly lower REE; lower health and activity scores in the quality of life questionnaires, and higher sleepiness scores.

Conclusions: Autonomic activity was similar in CRHO and control subjects. The degree of central adiposity correlated negatively with parasympathetic activity and positively with sympathetic activity in children with CRHO. These results provide a new perspective regarding autonomic balance in this unique patient population.
\end{abstract}

European Journal of Endocrinology 168 845-852

\section{Introduction}

Hypothalamic energy regulation is a complex system balancing energy intake, expenditure, and storage (1). Hypothalamic obesity (HO) can be caused by any type of hypothalamic impairment, either acquired or congenital (2). One such cause is tumors and their treatment effects, including craniopharyngioma (3). Craniopharyngioma tumor histology is benign and long-term survival rates are high $(4,5)$. Complications are also common, and both the tumor and the treatment can lead to significant long-term endocrinological and neurological morbidity. One of the most troublesome complications is HO (6). Hyperphagia is described in many cases and contributes to obesity; however, it was found that increased intake is not necessary for the development of HO (7). An imbalance in the activity of the autonomic nervous system (ANS) efferent hypothalamic pathways has been hypothesized as an important contributor to the development of this obesity $(1,2)$. A number of studies have suggested that decreased sympathetic activity $(8,9)$ and increased parasympathetic activity may play a role (10). Measurements included mostly biochemical markers of autonomic activity in small groups of patients. Confirming such an autonomic imbalance could lead to new treatment pathways for this type of obesity as well as contribute to the understanding of energy balance regulation in humans. Analysis of heart rate variability (HRV) is a simple and noninvasive technique that can be used to evaluate cardiovascular autonomic activity based on electrocardiography (ECG) recordings (11).

The objectives of this study were: i) to evaluate parasympathetic and sympathetic activity in children with craniopharyngioma-related $\mathrm{HO}$ (CRHO) compared with control subjects matched for sex, age, and BMI, using HRV indices as well as biochemical markers; ii) to assess relationships between markers of autonomic activity and the degree of obesity; and iii) to assess associations between the activity of the parasympathetic nervous system at the level of the pancreas, as 
indexed by pancreatic polypeptide, and insulin secretion. Vagal stimulation is the major regulator for pancreatic polypeptide secretion from pancreatic F cells (12), and plasma levels of pancreatic polypeptide are considered an indirect marker of parasympathetic activity on the pancreas. We hypothesized that children with CRHO will exhibit increased parasympathetic activity and decreased sympathetic activity compared with obese controls and that obesity will be positively related to parasympathetic activity and negatively to sympathetic activity in children with CRHO. We further hypothesized that pancreatic polypeptide levels will positively correlate with insulin secretion.

\section{Materials and methods}

Children and adolescents 8-20 years of age, overweight and obese, previously treated for craniopharyngioma, and followed at The Hospital for Sick Children in Toronto were invited to participate. Overweight and obesity were defined as BMI $\geq 85$ th and $\geq 95$ th percentile respectively for age on the gender-appropriate Centre for Disease Control growth curves (http://www. cdc.gov/growthcharts/clinical_charts.htm). Time from initial surgical treatment was at least 6 months. Known pituitary hormonal deficiencies were appropriately replaced before participation in the study. Control children with exogenous obesity were recruited from various outpatient clinics in the hospital as well as through volunteer flyers posted in the hospital. CRHO and control groups were matched for sex, age, and BMI. Exclusion criteria included individuals with other hypothalamic abnormalities or an underlying diagnosis of a cardiovascular abnormality. Patients taking medications that might affect autonomic activity were excluded or asked to hold the treatment for five half-lives before the day of the study visit. The study protocol was approved by the Research Ethics Board at The Hospital for Sick Children. All subjects and/or parents provided written informed consent for participation. In order to assess hypothalamic involvement in the CRHO subjects, brain imaging series were assessed for each (15 subjects had magnetic resonance images and one had computed tomography images). Hypothalamic involvement was classified using the grading system suggested by Müller et al. (3). Grade 0 includes tumors with no hypothalamic involvement/lesion. Grade 1 includes tumors with involvement of the anterior hypothalamus, the region posteriorly to the stalk and anteriorly to the mammillary bodies. Grade 2 includes tumors with hypothalamic involvement of the anterior and posterior hypothalamic area, i.e. involving the mammillary bodies and the area beyond mammillary bodies. Displacement of the mammillary bodies was not considered as involvement.

\section{Study protocol}

Evaluation included one visit to The Hospital for Sick Children followed by a 24-h urine collection and 24-h holter ECG monitoring completed at home. Subjects arrived fasting in the morning. Three measurements of height, weight, and waist circumference were completed for each subject using a standard, calibrated scale and wall-mounted stadiometer; the mean was taken for analyses. BMI (weight $(\mathrm{kg})) /\left(\right.$ height $\left.(\mathrm{m})^{2}\right)$ and the waist-to-height ratio were calculated for each subject. The waist-to-height ratio is a measure found to correlate with the degree of central obesity and with the risk for cardiometabolic complications in children (13). Three systolic and diastolic BP measurements taken 1 min apart in the right arm in the seated position were recorded; the mean of each was taken for analyses. Fasting blood-work was drawn for glucose, insulin, free thyroxine, and pancreatic polypeptide, as a marker of pancreatic parasympathetic activity. Body composition was assessed using air displacement plethysmography using the BOD POD (Life Measurement Incorporated, Concord, CA, USA) (14). Resting energy expenditure (REE) was measured by open-circuit indirect calorimetry using expired gas analysis for oxygen consumption and carbon dioxide elimination rates on a VMax Encore (VIASYS Healthcare, Dublin, OH, USA) (15).

\section{Questionnaires}

In order to assess health-related quality of life and fatigue, a common symptom in children with CRHO, participants completed the pediatric quality of life inventory generic core scale (PedsQL 4.0), the PedsQL multidimensional fatigue scale, and the Epworth sleepiness scale, a measure used to assess tendency to fall asleep during normal daily activities (M W Johns 1990-1997). Data regarding medical history were also collected. In order to detect symptoms suggesting autonomic dysfunction, questions regarding hot/cold spells, flushing, increased or decreased sweating, and swelling of hands and feet were included.

\section{Autonomic function}

To assess HRV, a five-lead holter ECG monitor (SEER Light and SEER Light Extend; GE Healthcare, Milwaukee, WI, USA) was placed. Subjects were asked to keep a journal of sleep and awake times during the recording period and were instructed to disconnect the device after $24 \mathrm{~h}$. HRV was analyzed by power spectral analysis of intervals between sequential $R$ waves (R-R intervals) (MARS GE Medical Systems); parameters were assessed over the full 24-h period, as well as separately for the awake and sleep periods. The power spectra were divided into very-low frequency (LF, <0.04 Hz), LF (0.04-0.15 Hz), and high frequency 
(HF, 0.15-0.4 Hz) bands based on accepted standards (11). Parasympathetic activity is best reflected by the HF component. The LF component reflects both sympathetic and parasympathetic activities and normalization of LF power or use of the $\mathrm{LF}: \mathrm{HF}$ ratio represents more reliably sympathetic cardiac activity (11). Normalized spectral band powers (in normalized units) were calculated as LFnu $=\mathrm{LF} /(\mathrm{LF}+\mathrm{HF})$ and $\mathrm{HFnu}=\mathrm{HF} /(\mathrm{LF}+\mathrm{HF})$. Urine was collected during a 24-h period for analysis of creatinine, catecholamine, and catecholamine metabolite levels, including homovanillic acid (HVA), vanillylmandelic acid (VMA), epinephrine, norepinephrine, metanephrine, and normetanephrine. The catecholamine-to-creatinine ratio was calculated for each. Levels of fasting plasma pancreatic polypeptide were measured using the Milliplex map Human Gut Hormone Panel (EMD Millipore, Darmstadt, Germany).

\section{Statistical analysis}

Statistical analysis was performed using the Statistical Package for Social Sciences Software (IBM SPSS Statistics, version 19.0). The sample size was based on mean values of HRV HFnu (reflecting parasympathetic activity) of $14.1 \pm 4.3$ in obese adolescents (16). With 14 subjects in each group, there is $80 \%$ power to detect a 1 s.D. difference between groups with an $\alpha$ of 0.05 . Continuous variables were assessed for normality by the Kolmogorov-Smirnov test and are expressed as mean \pm s.D. or median with 25 th and 75 th percentiles. Categorical variables are expressed as frequencies as well as proportions. Comparisons between groups were performed using two-tailed independent sample $t$-test or Mann-Whitney $U$ test for continuous variables and $\chi^{2}$ analysis for categorical parameters. Differences in these tests were considered statistically significant with $P \leq 0.05$. Pearson and/or Spearman univariate correlation analyses were performed for normally and non-normally distributed variables to assess measurements related to autonomic function and obesity.

\section{Results}

\section{Study subjects}

Sixteen out of 18 eligible children and adolescents with craniopharyngioma and 16 control subjects participated in the study. Subject characteristics are presented in Table 1. Mean age of CRHO patients was 16.5 \pm 3.4 years, mean BMI $36.4 \pm 9.5 \mathrm{~kg} / \mathrm{m}^{2}$, and mean time from diagnosis $7 \pm 3.7$ years (range 1.2-13.8). There were no differences between the groups in terms of age, gender, BMI, or ethnic origin. Mean waist-to-height ratio, fat mass, as well as fat-free mass were also not different between groups (Table 1). Fifteen out of
Table 1 Demographics and anthropometrics of the study population. Data are expressed as mean \pm s.D. or proportion (percentage). No significant difference in these variables was detected between groups.

\begin{tabular}{lll}
\hline Subject characteristic & CRHO & Control \\
\hline Females & $8 / 16(50 \%)$ & $8 / 16(50 \%)$ \\
Age (years) & $16.5 \pm 3.4$ & $15.6 \pm 2.1$ \\
Years from diagnosis & $7 \pm 3.7$ & \\
Caucasian & $10 / 16(63 \%)$ & $10 / 16(63 \%)$ \\
BMI $\left(\mathrm{kg} / \mathrm{m}^{2}\right)$ & $36.4 \pm 9.5$ & $35.9 \pm 8.5$ \\
Waist-to-height ratio & $0.64 \pm 0.1$ & $0.62 \pm 0.09$ \\
Fat mass $(\mathrm{kg})$ & $45.7 \pm 20.8$ & $42.4 \pm 20.9$ \\
Fat-free mass $(\mathrm{kg})$ & $52.1 \pm 12.5$ & $59.7 \pm 11.6$ \\
Hormonal deficiencies or supplementation & \\
Desmopressin & $13 / 16(81 \%)$ & 0 \\
Hydrocortisone & $13 / 16(81 \%)$ & 0 \\
GH & $10 / 16(63 \%)$ & 0 \\
Thyroxine & $13 / 16(81 \%)$ & $1 / 16(6 \%)$ \\
Any replacement & $15 / 16(94 \%)$ & $1 / 16(6 \%)$ \\
\hline
\end{tabular}

16 (94\%) CRHO subjects were receiving supplementation for pituitary deficiencies. Out of 13 patients with ACTH deficiency, ten were treated with GH and three had normal growth despite GH deficiency (GHD), a phenomenon described in this population (17). One of the control subjects was taking hormonal supplementation for autoimmune thyroiditis. All CRHO subjects had evidence for hypothalamic involvement on brain imaging, three subjects had grade 1 involvement, and 13 subjects had grade 2 involvement.

\section{Parasympathetic indices}

Indices of parasympathetic activity included HRV HF (normalized units and $\mathrm{ms}^{2}$ ), as well as plasma pancreatic polypeptide (Table 2, Fig. 1A). No significant differences were found between groups in HF during the full $24 \mathrm{~h}$, the sleep or awake periods. Fasting plasma pancreatic polypeptide levels were not different between groups and none of the pancreatic polypeptide levels were elevated above the normal range.

\section{Sympathetic indices}

Indices of sympathetic activity included HRV LF (normalized units) and LF/HF $\left(\mathrm{ms}^{2} / \mathrm{ms}^{2}\right)$ and urine catecholamines (Table 2, Fig. 1B). HRV sympathetic indices were not significantly different between groups for any of the periods tested. No significant difference in urine VMA or HVA and similar epinephrine and norepinephrine levels corrected for creatinine were detected. Urine metanephrine-to-creatinine levels were significantly higher in the CRHO group. HVA, epinephrine-to-creatinine, and total metanephrineto-creatinine ratios were normal in all study participants. Levels of urine VMA were mildly elevated in two CRHO subjects and four controls, and norepinephrineto-creatinine ratio was mildly elevated in two CRHO subjects and no controls. 
Table 2 Autonomic indices, measures of obesity, resting energy expenditure (REE), and questionnaire results. Continuous variables are presented as mean \pm s.D.s or median with interquartile range. Categorical data are presented as a proportion (\%).

\begin{tabular}{|c|c|c|c|}
\hline Variable & CRHO & Control & $P$ value \\
\hline Mean 24-h heart rate (beats/min) & $83 \pm 14$ & $83 \pm 9$ & 0.915 \\
\hline \multicolumn{4}{|l|}{ Parasympathetic indices } \\
\hline HRV 24-h HFnu & $0.36 \pm 0.10$ & $0.33 \pm 0.09$ & 0.412 \\
\hline HRV 24-h HF $\left(\mathrm{ms}^{2}\right)$ & $611 \pm 504$ & $459 \pm 336$ & 0.325 \\
\hline Fasting pancreatic polypeptide (pg/ml) & $30.5(18.2,72.4)$ & $41.2(28.5,115.3)$ & 0.597 \\
\hline \multicolumn{4}{|l|}{ Sympathetic indices } \\
\hline HRV 24-h LFnu & $0.98(0.95,0.98)$ & $0.97(0.96,0.98)$ & 0.273 \\
\hline HRV $24-h$ LF/HF $\left(\mathrm{ms}^{2} / \mathrm{ms}^{2}\right)$ & $1.62(1.37,2.41)$ & $1.89(1.44,2.99)$ & 0.650 \\
\hline U-NEpi/Creat (nmol/mmol) & $30.5 \pm 28.6$ & $18.3 \pm 7.2$ & 0.120 \\
\hline U-Epi/Creat (nmol/mmol) & $2.4 \pm 3.2$ & $1.1 \pm 0.5$ & 0.160 \\
\hline U-VMA & $16.1(14.5,23.7)$ & $19 . \overline{6}(17.4,31.9)$ & 0.250 \\
\hline U-NMet/Creat $(\mu \mathrm{mol} / \mathrm{mmol})^{*}$ & $0.14 \pm 0.07$ & $0.07 \pm 0.02$ & 0.004 \\
\hline U-Met/Creat $(\mu \mathrm{mol} / \mathrm{mmol})^{*}$ & $0.02 \pm 0.012$ & $0.01 \pm 0.01$ & 0.040 \\
\hline REE (kcal/day)* & $1679 \pm 308$ & $2050 \pm 323$ & 0.002 \\
\hline \multicolumn{4}{|l|}{ Questionnaires } \\
\hline PedsQL total score & $65.5 \pm 19.6$ & $75.2 \pm 17.5$ & 0.152 \\
\hline PedsQL physical health* & $64.0 \pm 23.4$ & $79.9 \pm 19.4$ & 0.046 \\
\hline Epworth sleepiness scale* & $10.2 \pm 3.6$ & $6.8 \pm 5.3$ & 0.045 \\
\hline PedsQL fatigue scale & $62.7 \pm 20.7$ & $68.7 \pm 22.2$ & 0.440 \\
\hline Symptoms suggesting autonomic dysregulation & $8 / 16(50 \%)$ & $3 / 16(19 \%)$ & 0.063 \\
\hline
\end{tabular}

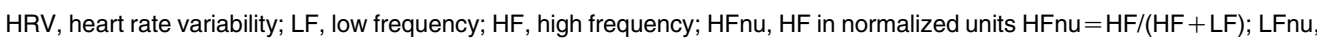
$\mathrm{LF}$ in normalized units LFnu $=\mathrm{LF} /(\mathrm{HF}+\mathrm{LF})$; U-NE/creatinine, urine norepinephrine-to-creatinine ratio; U-Epi/Creat, urine epinephrine-to-creatinine ratio; U-VMA, urine vanillylmandelic acid; U-Met/creatinine, urine metanephrine-to-creatinine ratio; U-NMet/creatinine, urine normetanephrine-to-creatinine ratio. ${ }^{*} P<0.05 t$-test (two tailed).

\section{Questionnaires and REE}

Craniopharyngioma subjects scored lower in the physical health section of the PedsQL questionnaires (Table 2). There was no significant difference between groups in the total score on the PedsQL or in other sections of the questionnaire. CRHO subjects scored higher on the Epworth sleepiness scale. A trend toward a higher proportion of CRHO subjects had at least one symptom suggesting autonomic dysfunction. REE was significantly lower in the CRHO group.

\section{Correlation between autonomic indices and measures of obesity}

In the CRHO group, HRV parasympathetic indices during the full 24-h recording as well as during awakeness correlated negatively with the waistto-height ratio $(r=-0.53, P=0.034$ and $r=-0.55$, $P=0.029$ respectively; Fig. 2). HRV sympathetic indices (LF/HF) during the full 24-h recording and during sleep correlated positively with the waistto-height ratio $(r=0.51, P=0.043$ and $r=0.54$, $P=0.033$ respectively). In the control group, similar relationships between adiposity and parasympathetic markers were identified $(r=-0.54, P=0.029$ over $24 \mathrm{~h}$ and $r=-0.52, P=0.037$ during awakeness); sympathetic markers on the other hand did not correlate with the degree of obesity. The plasma pancreatic polypeptide levels did not correlate with fasting insulin levels in either group.

\section{Discussion}

In this study, children and adolescents with HO following treatment for craniopharyngioma exhibited similar levels of sympathetic and parasympathetic activity based on HRV indices, plasma pancreatic polypeptide, and urine epinephrine, norepinephrine, and VMA compared with obese controls. Using prolonged ECG recording for HRV analysis enabled us to evaluate autonomic activity while subjects were engaging in routine daily activities. To the best of our knowledge, this is the first study to investigate HRV in youth with CRHO, providing a new perspective on ANS function in this population.

The ANS is known to have three divisions, the sympathetic, parasympathetic, and enteric. The relationships between the enteric nervous system and the CNS are complex, involving the two other ANS divisions (18). Various methods can be used for assessing autonomic activity; most rely on evaluation of cardiovascular reflexes indirectly reflecting sympathetic or parasympathetic activity (19). Other indirect measures can include levels of peptides involved in energy regulation as a surrogate of the autonomic effect on the enteric system. As no single test accurately reflects the function of a specific branch of the ANS, batteries of tests may be used. We chose to combine methods assessing biochemical markers of autonomic activity and a prolonged 24-h ECG recording. This recording enabled analysis of autonomic activity over the full $24 \mathrm{~h}$ as well as separately for awake and sleep 

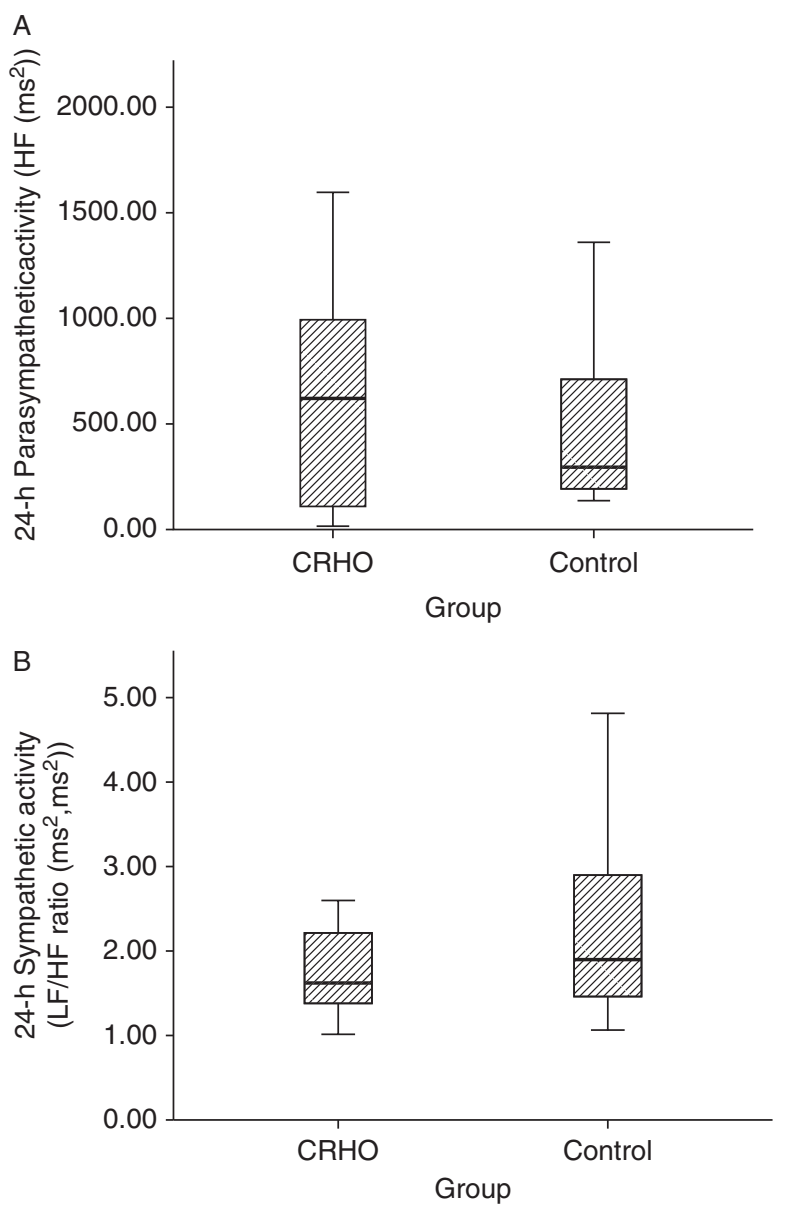

Figure 1 Parasympathetic (A) and sympathetic (B) HRV indices in the two study groups. The median, upper, and lower quartiles and the range are presented. No significant difference in parasympathetic (HF power $\left(\mathrm{ms}^{2}\right)$ ) or sympathetic (LF:HF ratio) markers was found between the groups.

periods. HRV is a simple, noninvasive method for assessing cardiac autonomic activity with good reproducibility in children (20). HRV methods, particularly the power spectral analysis of the frequency domain, are gaining wider use in clinical research involving various pediatric and adult populations (21).

There is a clear link between hypothalamic damage and altered ANS balance in experimental animals; rats with lesions of the ventromedial and lateral hypothalamus are characterized by hyperphagia, reduced sympathetic and increased parasympathetic activities, delayed satiety, decreased thermogenesis, and decreased ability to mobilize fatty acids. These rats develop obesity, even with food restriction $(22,23)$. The literature suggests a relationship between autonomic dysfunction and $\mathrm{HO}$ in craniopharyngioma patients, yet data in this area are sparse and the evidence holds some important limitations. Two studies that assessed biochemical markers of autonomic activity support decreased sympathetic activity in craniopharyngioma patients
$(8,9)$. When compared with our findings, differences in the characteristics of the control population (i.e. healthy children with short stature as control subjects vs obese in our study) and a variety of methods of measurement may account for the discrepancies. Further support for the role of sympathetic impairment comes from the improvement seen in terms of reduced weight gain and increased activity and attention when treating CRHO patients with medications that increase sympathetic tone $(24,25,26)$. However, while a beneficial effect was demonstrated, when compared with subjects with other causes of obesity, the CRHO patients actually showed partial resistance to the medication and a milder response (25). In support of increased parasympathetic activity, elevated levels of insulin have been reported in CRHO, even after adjusting for fat mass $(27,28)$, and improvement in terms of reduced weight gain was demonstrated with treatments targeted at suppressing pancreatic $\beta$-cell activity $(10,29,30)$. As these are indirect measures that most likely are affected by multiple factors, their interpretation is limited. In adults with untreated GHD, HRV analyses suggest decreased sympathetic and increased parasympathetic activity compared with healthy controls (31). Assuming that similar trends would occur in children, those with untreated GHD would have further increased differences between our study and control groups. In this study, the number of children with GHD and untreated $(n=3)$ was too small to analyze.

We recruited patients at least 6 months following the initial treatment for craniopharyngioma in order to assess them in a more stable clinical state, when pituitary imbalances are adequately treated and patients are engaged in routine activities. Rapid weight gain is known to begin very early after treatment (17), and assessment of autonomic activity earlier in the postoperative period might have allowed us to better differentiate the tumor and treatment autonomic effects vs those of obesity per se.

A negative correlation between HRV parasympathetic indices and central obesity is demonstrated in both of our study groups. Similar relationships are described in the literature on childhood obesity, with lower HF power on HRV analysis (a parasympathetic marker) in obese children compared with normal-weight children (32, 33). A positive correlation between the degree of obesity and the HRV sympathetic markers is found only in the CRHO group. The literature on this topic in children with obesity is conflicting, with studies demonstrating increased (33), reduced (32), or similar (34) levels of sympathetic activity in obese children when compared with normal-weight children.

Fasting pancreatic polypeptide levels were similar in CRHO and control subjects; one potential explanation is that clear differences in levels might be present only in the stimulated state, in response to food intake. A surprising finding is the increased urine-free metanephrines in CRHO subjects compared with controls, 

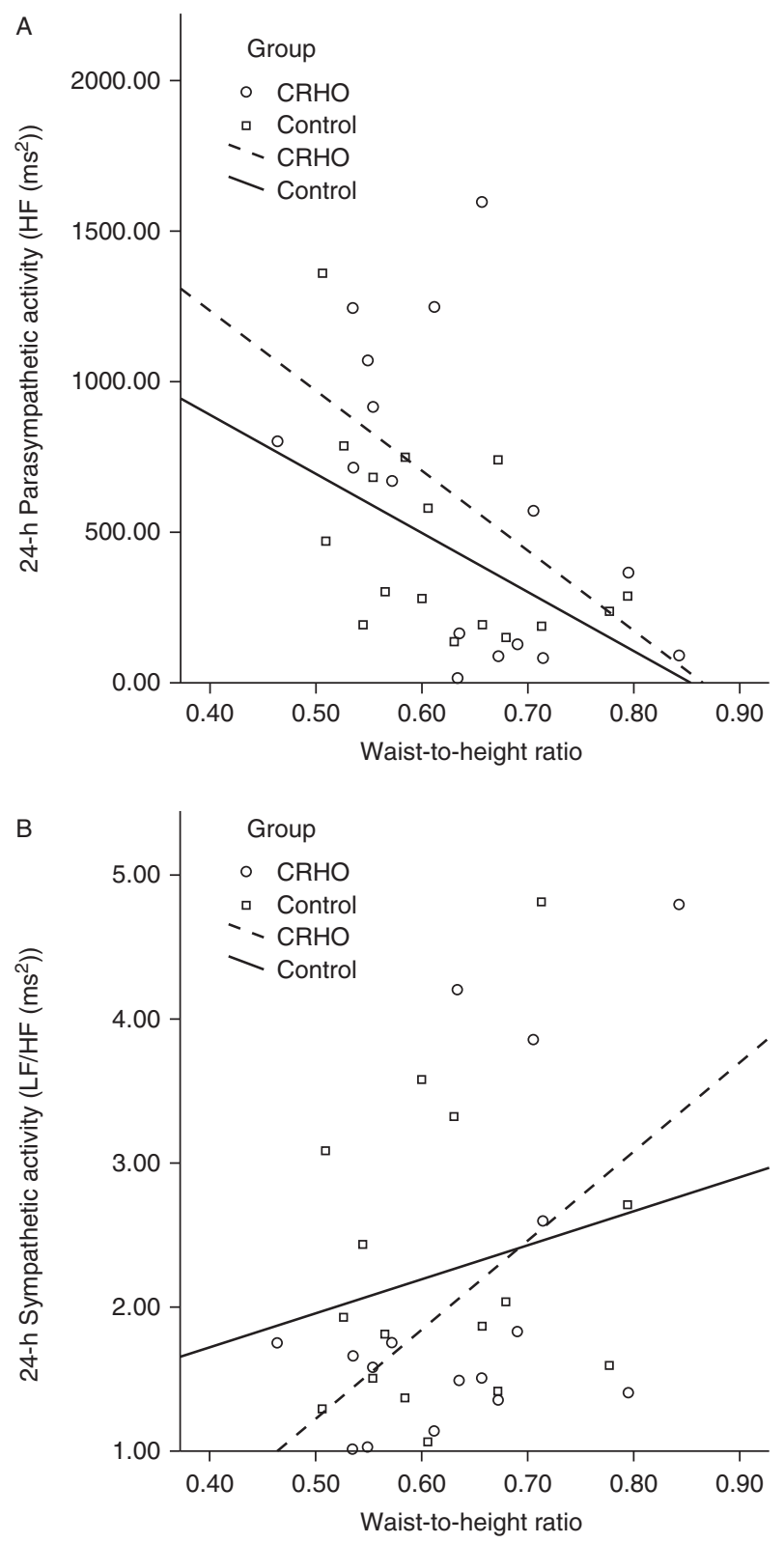

Figure 2 Heart rate variability autonomic indices and the degree of central obesity. (A) Twenty-four hour parasympathetic activity (HF $\left(\mathrm{ms}^{2}\right)$ ) vs waist-to-height ratio. A negative correlation between adiposity and parasympathetic activity is demonstrated in both study groups. In the CRHO group, $r=-0.53, P=0.034$; in the control group, $r=-0.54, P=0.029$. (B) Twenty-four hour sympathetic activity (logLF/HF $\left(\mathrm{ms}^{2} / \mathrm{ms}^{2}\right)$ ) vs waist-to-height ratio. A significant negative correlation between adiposity and sympathetic activity is demonstrated only in the $\mathrm{CRHO}$ group, $r=0.51, P=0.043$.

despite similar levels of other catecholamines and their metabolites. As levels of total metanephrines were in the normal range in both groups, the clinical significance of this finding is not clear. Metanephrines are the product of catecholamine degradation in the adrenal glands; a potential explanation for this finding is that adrenal catecholamine production is higher in this population in order to compensate for decreased neuronal catecholamine secretion. Alternatively, this could reflect increased degradation of these metabolites and potentially a decreased local effect.

Subjects with CRHO demonstrated significantly lower REE, lower scores in the physical activity sections of the PedsQL, and higher sleepiness scores. We found the REE to be decreased in CRHO subjects despite no difference in sympathetic activity. This might not be surprising as although tonic sympathetic activity affects REE (35), this $\beta$-adrenergic support is relatively minor and it appears that the sympathetic system has a more significant role in the thermogenic effect of food. The two groups were similar in terms of fat-free mass and tests were conducted under similar conditions; therefore, these could not explain the lower REE in CRHO. These differences between groups are supported by previous studies in which patients with craniopharyngioma were found to have lower REE compared with controls, a finding not explained by differences in body composition $(7,36)$. The decreased habitual daily physical activity may be contributing to lower REE in patients with craniopharyngioma. Other, potentially central mechanisms, may also be involved in contributing to low REE in this population. Decreased quality of life (37), sleep fragmentation, and increased daytime sleepiness have also been described in this population $(4,38)$.

There are limitations to our study. A relatively small sample size secondary to the rarity of this condition might have altered the ability to detect small differences between CRHO patients and controls, despite an adequate power calculation. A control group of normal-weight subjects was not included in this study; therefore, we cannot draw direct conclusions as to how autonomic measures in the CRHO group would differ from those of normal-weight subjects. However, given that this study was designed to test differences between autonomic function and HO vs nonhypothalamic obesity, we felt this was the most appropriate 'control' group for comparison. The cross-sectional design of the study does not allow for assessment of causality. Another challenging issue is that alimentary autonomic activity cannot be evaluated directly; therefore, we must rely on the reflection of this activity in the cardiovascular markers. This may leave important differences between groups unrecognized. Nutrient intake is related to cardiac autonomic function; in obese adults cardiac parasympathetic dysfunction was found to be associated with higher carbohydrate intake and lower fat and protein intake (39). Whether differences in nutrient intake may have 'masked' differences in autonomic activity could not be determined and would be interesting to include in future studies.

In summary, energy expenditure is regulated by a complex interplay between hormonal, neuronal, and nutrient signals involving, among other systems, the 
three ANS divisions and the hypothalamus. Isolating the specific role of each variable is subject to multiple limitations. While our findings do not support our original hypothesis, they do provide additional information regarding autonomic activity in the CRHO population. Future research evaluating autonomic activity in craniopharyngioma patients prospectively from the time of diagnosis following alterations in autonomic activity and weight could add valuable information as to how these systems influence each other in this population. Additional study of the relationships between autonomic activity and fat distribution would be of value.

\section{Declaration of interest}

The authors declare that there is no conflict of interest that could be perceived as prejudicing the impartiality of the research reported.

\section{Funding}

This study was supported by a b.r.a.i.n.child research grant. M Cohen was supported by a Canadian Pediatric Endocrine Group (CPEG) fellowship grant. C Syme was supported by a fellowship from the Heart and Stroke Foundation of Ontario.

\section{Acknowledgements}

The authors thank the SickKids Physiological Research Unit staff as well as the participants and their families for the time and effort invested in the study.

\section{References}

1 Lustig RH. Hypothalamic obesity: the sixth cranial endocrinopathy. Endocrinologist 200212 8. (doi:10.1097/00019616-200 205000-00008)

2 Hochberg I \& Hochberg Z. Expanding the definition of hypothalamic obesity. Obesity Reviews 201011 709-721. (doi:10.1111/ j.1467-789X.2010.00727.x)

3 Müller HL, Gebhardt U, Teske C, Faldum A, Zwiener I, WarmuthMetz M, Pietsch T, Pohl F, Sorensen N \& Calaminus G. Post-operative hypothalamic lesions and obesity in childhood craniopharyngioma: results of the multinational prospective trial KRANIOPHARYNGEOM 2000 after 3-year follow-up. European Journal of Endocrinology 2011165 17-24. (doi:10.1530/EJE-11-0158)

4 Poretti A, Grotzer M, Ribi K, Schonle E \& Boltshauser E. Outcome of craniopharyngioma in children: long-term complications and quality of life. Developmental Medicine and Child Neurology 200446 220-229. (doi:10.1111/j.1469-8749.2004.tb00476.x)

5 Visser J, Hukin J, Sargent M, Steinbok P, Goddard K \& Fryer C. Late mortality in pediatric patients with craniopharyngioma. Journal of Neuro-Oncology $2010 \mathbf{1 0 0}$ 105-111. (doi:10.1007/ s11060-010-0145-5)

6 Sughrue M, Yang I, Kane A, Fang S, Clark A, Aranda D, Barani I \& Parsa A. Endocrinologic, neurologic, and visual morbidity after treatment for craniopharyngioma. Journal of Neuro-Oncology 2011 101 463-476. (doi:10.1007/s11060-010-0265-y)

7 Holmer H, Pozarek G, Wirfalt E, Popovic V, Ekman B, Bjork J \& Erfurth EM. Reduced energy expenditure and impaired feedingrelated signals but not high energy intake reinforces hypothalamic obesity in adults with childhood onset craniopharyngioma. Journal of Clinical Endocrinology and Metabolism 201095 53955402. (doi:10.1210/jc.2010-0993)
8 Coutant R, Maurey H, Rouleau S, Mathieu E, Mercier P, Limal JM \& Le Bouil A. Defect in epinephrine production in children with craniopharyngioma: functional or organic origin? Journal of Clinical Endocrinology and Metabolism $2003 \mathbf{8 8}$ 5969-5975. (doi:10.1210/jc.2003-030552)

9 Roth CL, Hunneman DH, Gebhardt U, Stoffel-Wagner B, Reinehr T \& Müller HL. Reduced sympathetic metabolites in urine of obese patients with craniopharyngioma. Pediatric Research 200761 496-501. (doi:10.1203/pdr.0b013e3180332cd6)

10 Lustig RH, Rose SR, Burghen GA, Velasquez-Mieyer P, Broome DC, Smith K, Li H, Hudson MM, Heideman RL \& Kun LE. Hypothalamic obesity caused by cranial insult in children: altered glucose and insulin dynamics and reversal by a somatostatin agonist. Journal of Pediatrics 1999135 162-168. (doi:10.1016/ S0022-3476(99)70017-X)

11 Heart rate variability: standards of measurement, physiological interpretation and clinical use. Task Force of the European Society of Cardiology and the North American Society of Pacing and Electrophysiology. Circulation 199693 1043-1065. (doi:10.1161/01.CIR.93.5.1043)

12 Schwartz TW, Holst JJ, Fahrenkrug J, Jensen SL, Nielsen OV, Rehfeld JF, de Muckadell OB \& Stadil F. Vagal, cholinergic regulation of pancreatic polypeptide secretion. Journal of Clinical Investigation 197861 781-789. (doi:10.1172/JCI108992)

13 Jasmeet S, Mokha SRS, Dasmahapatra P, Fernandez C, Chen W, $\mathrm{Xu} \mathrm{J} \mathrm{\&} \mathrm{Berenson} \mathrm{GS.} \mathrm{Utility} \mathrm{of} \mathrm{waist-to-height} \mathrm{ratio} \mathrm{in} \mathrm{assessing} \mathrm{the}$ status of central obesity and related cardiometabolic risk profile among normal weight and overweight/obese children: The Bogalusa Heart Study. BMC Pediatrics 201010 73. (doi:10. 1186/1471-2431-10-73)

14 Dempster P \& Aitkens S. A new air displacement method for the determination of human body composition. Medicine and Science in Sports and Exercise 199527 1692-1697. (doi:10.1249/ 00005768-199512000-00017)

15 Pencharz PB \& Azcue MP. Measuring resting energy expenditure in clinical practice. Journal of Pediatrics 1995127 269-271. (doi:10.1016/S0022-3476(95)70306-3)

16 Martini G, Riva P, Rabbia F, Molini V, Ferrero GB, Cerutti F, Carra R \& Veglio F. Heart rate variability in childhood obesity. Clinical Autonomic Research 200111 87-91. (doi:10.1007/ BF02322051)

17 Müller HL, Bueb K, Bartels U, Roth C, Harz K, Graf N, Korinthenberg R, Bettendorf M, Kuhl J, Gutjahr P et al. Obesity after childhood craniopharyngioma - German multicenter study on pre-operative risk factors and quality of life. Klinische Pädiatrie 2001213 244-249. (doi:10.1055/s-2001-16855)

18 Mayer EA. Gut feelings: the emerging biology of gut-brain communication. Nature Reviews. Neuroscience 2011 12 453-466. (doi:10.1038/nrn3071)

19 Zygmunt A \& Stanczyk J. Methods of evaluation of autonomic nervous system function. Archives of Medical Science 20106 11-18. (doi:10.5114/aoms.2010.13500)

20 Dietrich A, Rosmalen JG, Althaus M, van Roon AM, Mulder LJ, Minderaa RB, Oldehinkel AJ \& Riese H. Reproducibility of heart rate variability and baroreflex sensitivity measurements in children. Biological Psychology 201085 71-78. (doi:10.1016/ j.biopsycho.2010.05.005)

21 Rajendra Acharya U, Paul Joseph K, Kannathal N, Lim CM \& Suri JS. Heart rate variability: a review. Medical $\mathcal{E}$ Biological Engineering \& Computing 200644 1031-1051. (doi:10.1007/ s11517-006-0119-0)

22 Bray GA, Inoue S \& Nishizawa Y. Hypothalamic obesity. The autonomic hypothesis and the lateral hypothalamus. Diabetologia 198120 (Suppl) 366-377. (doi:10.1007/BF00254505)

23 Sakaguchi T, Arase K \& Bray GA. Sympathetic activity and food intake of rats with ventromedial hypothalamic lesions. International Journal of Obesity 198812 285-291.

24 Mason PW, Krawiecki N \& Meacham LR. The use of dextroamphetamine to treat obesity and hyperphagia in children treated for craniopharyngioma. Archives of Pediatrics \& Adolescent Medicine 2002156 887-892. (doi:poa10446[pii]) 
25 Danielsson P, Janson A, Norgren S \& Marcus C. Impact sibutramine therapy in children with hypothalamic obesity or obesity with aggravating syndromes. Journal of Clinical Endocrinology and Metabolism 200792 4101-4106. (doi:10.1210/jc. 2007-0826)

26 Schultes B, Ernst B, Schmid F \& Thurnheer M. Distal gastric bypass surgery for the treatment of hypothalamic obesity after childhood craniopharyngioma. European Journal of Endocrinology 2009161 201-206. (doi:10.1530/EJE-09-0079)

27 Simoneau-Roy J, O'Gorman C, Pencharz P, Adeli K, Daneman D \& Hamilton J. Insulin sensitivity and secretion in children and adolescents with hypothalamic obesity following treatment for craniopharyngioma. Clinical Endocrinology $2010 \mathbf{7 2}$ 364-370. (doi:10.1111/j.1365-2265.2009.03639.x)

28 Roth CL, Gebhardt U \& Müller HL. Appetite-regulating hormone changes in patients with craniopharyngioma. Obesity 201119 36-42. (doi:10.1038/oby.2010.80)

29 Hamilton JK, Conwell LS, Syme C, Ahmet A, Jeffery A \& Daneman D. Hypothalamic obesity following craniopharyngioma surgery: results of a pilot trial of combined diazoxide and metformin therapy. International Journal of Pediatric Endocrinology 20112011 417949. (doi:10.1155/2011/417949)

30 Lustig RH, Hinds PS, Ringwald-Smith K, Christensen RK, Kaste SC, Schreiber RE, Rai SN, Lensing SY, Wu S \& Xiong X. Octreotide therapy of pediatric hypothalamic obesity: a doubleblind, placebo-controlled trial. Journal of Clinical Endocrinology and Metabolism 200388 2586-2592. (doi:10.1210/jc.2002030003)

31 Leong KS, Mann P, Wallymahmed M, MacFarlane IA \& Wilding JP. Abnormal heart rate variability in adults with growth hormone deficiency. Journal of Clinical Endocrinology and Metabolism 2000 85 628-633. (doi:10.1210/jc.85.2.628)

32 Vanderlei LC, Pastre CM, Freitas Junior IF \& Godoy MF. Analysis of cardiac autonomic modulation in obese and eutrophic children. Clinics 201065 789-792. (doi:10.1590/S1807-593220100008 00008)
33 Rodriguez-Colon SM, Bixler EO, Li X, Vgontzas AN \& Liao D. Obesity is associated with impaired cardiac autonomic modulation in children. International Journal of Pediatric Obesity 20116 128-134. (doi:10.3109/17477166.2010.490265)

34 Yakinci C, Mungen B, Karabiber H, Tayfun M \& Evereklioglu C. Autonomic nervous system functions in obese children. Brain $\mathcal{E}$ Development 200022 151-153. (doi:10.1016/S0387-7604(00) 00094-2)

35 Monroe MB, Seals DR, Shapiro LF, Bell C, Johnson D \& Parker Jones P. Direct evidence for tonic sympathetic support of resting metabolic rate in healthy adult humans. American Journal of Physiology. Endocrinology and Metabolism 2001280 E740-E744.

36 Kim RJ, Shah R, Tershakovec AM, Zemel BS, Sutton LN, Grimberg A \& Moshang T. Energy expenditure in obesity associated with craniopharyngioma. Child's Nervous System 201026 913-917. (doi:10.1007/s00381-009-1078-1)

37 Rakhshani N, Jeffery AS, Schulte F, Barrera M, Atenafu EG \& Hamilton JK. Evaluation of a comprehensive care clinic model for children with brain tumor and risk for hypothalamic obesity. Obesity 201018 1768-1774. (doi:10.1038/oby.2009.491)

38 Lipton J, Megerian JT, Kothare SV, Cho YJ, Shanahan T, Chart H, Ferber R, Adler-Golden L, Cohen LE, Czeisler CA et al. Melatonin deficiency and disrupted circadian rhythms in pediatric survivors of craniopharyngioma. Neurology 200973 323-325. (doi:10. 1212/WNL.Ob013e3181af78a5)

39 Valensi P, Paries J, Lormeau B, Attia S \& Attali JR. Influence of nutrients on cardiac autonomic function in nondiabetic overweight subjects. Metabolism 200554 1290-1296. (doi:10.1016/ j.metabol.2005.04.016)

Received 14 December 2012

Revised version received 8 February 2013

Accepted 12 March 2013 